\title{
Spin and mass content of linearized Poincaré gauge theories
}

\author{
ERIC A LORD and K P SINHA \\ Department of Applied Mathematies, Indian Institute of Science, \\ Bangalore 560012, India
}

MS received 12 January 1988; revised 22 March 1988

\begin{abstract}
A unified gauge theory of massless and massive spin-2 fields is of considerable current interest. The Poincare gauge theories with quadratic Lagrangian are linearized, and the conditions on the parameters are found which will lead to viable linear theories with massive gauge particles. As well as the $2^{+}$massless gravitons coming from the translational gauge potential, the rotational gauge potentials, in the linearized limit, give rise to $2^{+}$and $2^{-}$ particles of equal mass, as well as a massive pseudoscalar.
\end{abstract}

Keywords. Gravitation; gauge theortes; teleparallelism: rotons.

PACS No. $11 \cdot 15$

\section{Introduction}

There has been considerable inierest in gauge theories of massless and massive spin-2 fields, in the recent past (Sinha 1984). Here we investigate the spin-two gauge fields of Poincare gauge theory (Hehl 1980). In this theory the gauge potentials are a tetrad $e_{i}^{\alpha}$ and an anholonomic connection $\Gamma_{i}^{\alpha \beta}=-\Gamma_{i}^{\beta \alpha}$. The gauge field strengths are torsion and curvature, defined by

$$
\begin{aligned}
& F_{i j}{ }^{\beta}=\partial_{i} e_{j}^{\beta}-\partial_{j} e_{i}^{\beta}-e_{i}^{\gamma \gamma} \Gamma_{j i^{\beta}}{ }^{\beta}+e_{j}^{\gamma} \Gamma_{i \gamma}{ }^{\beta}, \\
& F_{i j a}{ }^{\beta}=\partial_{i} \Gamma_{j a}{ }^{\beta}-\gamma_{j} \Gamma_{i x}{ }^{\beta}-\Gamma_{i \alpha^{j}} \Gamma_{j j}{ }^{\beta}+\Gamma_{j x^{i}} \Gamma_{j j}{ }^{\beta} .
\end{aligned}
$$

It is convenient to define the following contractions of the curvature and torsion:

$$
F_{\alpha}=F_{\alpha \beta}{ }^{\beta}, \quad F_{\alpha \beta}=F_{\gamma \alpha \beta}{ }^{\gamma}, \quad H=F_{\alpha}{ }^{\alpha} .
$$

One can form three invariants quadratic in torsion (i.e. invariant under general coordinate transformations and spacetime-dependent Lorentz rotations of the tetrad):

$$
\left.\begin{array}{l}
J_{1}=F_{\alpha \beta \gamma} F^{\alpha \beta \gamma}, \quad J_{2}=F_{\alpha \beta \gamma} F^{\alpha i \beta}, \\
J_{3}=F_{\alpha} F^{\alpha} .
\end{array}\right\}
$$

One can form six invariants quadratic in curvature:

$$
\left.\begin{array}{l}
I_{1}=F_{\alpha \beta \gamma \delta} F^{\alpha \beta \gamma \delta} ; \quad I_{2}=F_{\alpha \beta \gamma \gamma} F^{\gamma \gamma \delta \beta}, \\
I_{3}=F_{\alpha \beta \gamma \delta} F^{\gamma \delta \alpha \beta} ; \quad I_{4}=F_{\alpha \beta} F^{\beta \alpha}, \\
I_{5}=F_{\alpha \beta} F^{\alpha \beta \beta} ; \quad I_{6}=F^{2} .
\end{array}\right\}
$$


The most general Lagrangian density leading to Euler Lagrange equations linear in the highest (second) order derivatives of the potentials, is

$$
\begin{aligned}
\mathscr{V}= & e\left(a_{1} I_{1}+a_{2} I_{2}+a_{3} I_{3}+a_{4} I_{4}+a_{5} I_{5}+a_{6} I_{6}\right. \\
& \left.+b F+c_{1} J_{1}+c_{2} J_{2}+c_{3} J_{3}+\Lambda\right),
\end{aligned}
$$

where the $a^{\prime}$ s, $b$, the $c^{\prime}$ s and $\Lambda$ are constants.

According to the extended Gauss-Bonnet theorem (Nieh 1980), the combination

$$
e\left(I_{3}-4 I_{4}+I_{6}\right)
$$

is a divergence; this fact could be used to eliminate one of the terms in $\mathscr{V}$, but it is not convenient to do so. The theorem implies that a particular version of Poincaré gauge theory is unaltered by changing the parameters according to

$$
a_{3} \rightarrow a_{3}+\alpha, \quad a_{4} \rightarrow a_{4}-4 \alpha, \quad a_{6} \rightarrow a_{6}+\alpha .
$$

For simplicity, we shall consider only the theories with vanishing cosmological constant, $\Lambda=0$.

The purpose of this work is to discover the conditions that the coefficients in (6) must satisfy in order that the linearized version of a Poincare gauge theory shall have the following properties:

(i) the irreducible parts of the potentials shall, in the linearised limit, describe decoupled modes with well-defined spins

(ii) tachyons shall be absent

(iii) ghosts shall be absent (condition of positive energy).

For simplicity we shall consider only the case of vanishing cosmological constant $(\Lambda=0)$. When $\Lambda \neq 0$ the linearization on a flat background is no longer appropriate and one has to consider perturbations on a de Sitter background. It was shown by Lord et al (1974) that the perturbations on a de Sitter background, in the context of Einstein's theory, describe a massive spin-2 field and also a massive spin-zero field.

\section{The teleparallelism limit}

The restricted version of the theory, in which curvature vanishes, is referred to as the teleparallelism limit. The reference system can in this case be chosen so that the anholonomic connection vanishes (Hehl 1980), so that the torsion becomes simply the 'object of anholonimity'

$$
F_{i j}^{\alpha}=\partial_{i} e_{j}^{\alpha}-\partial_{j} e_{i}^{\alpha} \text {. }
$$

The teleparallelism limit of Poincare gauge theory is expected to describe classical gravitation. In particular, the teleparallelism theory given by

$$
y^{\prime}=-\left(e / 4 l^{2}\right)\left(-\frac{1}{2} J_{1}-J_{2}+2 J_{3}\right)
$$

is Einstein's general relativity (Hehl 1980; Cho 1976). 
The teleparallelism theories can be linearized by considering small perturbations on a flat spacetime. That is, we write

$$
e_{i}^{\alpha}=\delta_{i}^{\alpha}+\phi_{i}^{\alpha}
$$

and retain only first-order terms in the small quantities $\phi_{i}^{\alpha}$. The distinction between Latin (holonomic) and Greek (anholonomic) indices vanishes in this approximation, and the orthonomality of the tetrad implies

$$
\phi_{\alpha \beta}=\phi_{\beta \alpha}
$$

We have, now,

$$
F_{\alpha \beta \gamma}=\partial_{\alpha} \phi_{\beta \gamma}-\partial_{\beta} \phi_{\alpha \gamma}, \quad F_{\alpha}=\partial_{\alpha} \phi-\partial^{\beta} \phi_{\beta \alpha}
$$

where $\phi=\phi_{\alpha}^{\alpha}$. The linearized Euler-Lagrange equations for $\phi_{\alpha \beta}$ are

$$
\partial_{\alpha} K^{\alpha(\beta \gamma)}=0
$$

where

$$
K^{\alpha \beta \gamma}=\left(2 c_{1}+c_{2}\right)\left(\partial^{\alpha} \phi^{\beta \gamma}-\partial^{\beta} \phi^{\alpha \gamma}\right)+c_{3}\left(F^{\alpha} \eta^{\beta \gamma}-F^{\beta} \eta^{\alpha \gamma}\right)
$$

Contracting on $\beta \gamma$ gives

$$
\left(2 c_{1}+c_{2}+3 c_{3}\right)\left(\square \phi-\partial^{\alpha \beta} \phi_{\alpha \beta}\right)=0 .
$$

Therefore, provided that $2 c_{1}+c_{2}+3 c_{3} \neq 0$,

$$
\square \phi=\partial^{\alpha \beta} \phi_{\alpha \beta}
$$

Substituting this expression back into the field equations gives

$$
\left(2 c_{1}+c_{2}\right)\left(\square \phi^{\beta \gamma}-\partial^{(\beta} \partial_{\alpha} \phi^{\gamma) \alpha}\right)+c_{3}\left(\partial^{(\beta} \partial_{\alpha} \phi^{\gamma) \alpha}-\partial^{\beta \gamma} \phi\right)=0 .
$$

These equations become the equation for a massless spin-2 field provided we require them to be invariant under the gauge transformation

$$
\phi_{\alpha \beta} \rightarrow \partial_{\alpha} \xi_{\beta}+\partial_{\beta} \xi_{\alpha}
$$

This requirement is satisfied provided

$$
2 c_{1}+c_{2}+c_{3}=0
$$

The Lagrangian density for the full nonlinear teleparallelism theory is then of the form

$$
\mathscr{V}=-\frac{e}{4 l^{2}}\left(-\frac{1}{2}(\lambda+1) J_{1}+(\lambda-1) J_{2}+2 J_{3}\right)
$$

where $\lambda$ is a free parameter. These theories all have a Schwarzschild solution and coincide with Einstein's theory up to the fourth post-Newtonian approximation (Hehl 1980; Nitsch 1980). Einstein's theory is given by $\lambda=0$. The theory of Hehl and his coworkers has $\lambda=1$.

To avert misunderstanding, we remind the reader that, in a Poincare gauge theory, 
the metric and the connection are independent dynamical fields. In the teleparallelism limit, the curvature tensor (2), constructed from the connection, vanishes. This tensor should not be confused with the Riemann tensor, constructed from the metric and its derivatives by way of the Christoffel symbols.

The appropriateness of linearizing on a (metrically!) flat background depends on the vanishing of the cosmological term. If the cosmological constant is not zero, one has to consider perturbations on a de Sitter background. It was shown by Lord et al (1974) that the perturbations on a de Sitter background in Einstein's theory describe a massive spin-2 field and also a massive spin-zero field.

\section{The roton sector}

In order to study the roton sector, we consider a different limit. We retain the dynamical freedom of the connection, but freeze the tetrad by imposing the constraint

$$
e_{i}^{\alpha}=\delta_{i}^{\alpha}
$$

We now have a theory of a field $\Gamma_{v \alpha \beta}$ in Minkowski space. Decompose this tensor field into its irreducible parts, writing

$$
\Gamma_{\alpha \beta \gamma}=\psi_{\alpha \beta \gamma}+\chi_{\alpha \beta \gamma}+\frac{2}{3} \eta_{\alpha[\beta \gamma]}
$$

where

$$
A_{\gamma}=\Gamma_{\alpha \gamma}^{\alpha}, \quad \chi_{\alpha \beta \gamma}=\Gamma_{[\alpha \beta \gamma]},
$$

and $\psi_{\alpha \beta y}$ satisfies the following identities:

$$
\left.\begin{array}{l}
\psi_{\alpha \beta \gamma}=-\psi_{\alpha \gamma \beta}, \quad \psi_{\alpha \gamma}^{\alpha}=0, \\
\psi_{\alpha \beta \gamma}+\psi_{\beta \gamma \alpha}+\psi_{\gamma \alpha \beta}=0 .
\end{array}\right\}
$$

The dual of $\psi_{\alpha \beta \gamma}, \tilde{\psi}_{\alpha \beta \gamma}=\frac{1}{2} \varepsilon_{\beta \gamma \mu \nu} \psi_{\alpha}^{\mu \nu}$ satisfies the same identities. The trace $A_{\alpha}$, the completely skew-symmetric part $\chi_{\alpha \beta \gamma}$, and the self-dual and anti-self-dual parts of $\psi_{\alpha \beta \gamma}=$ $-\psi_{\alpha \beta \gamma}$ are the four irreducible parts of the Lorentz tensor $\Gamma_{\alpha \beta \gamma}=-\Gamma_{\alpha \gamma \beta}$.

It is convenient to introduce the dual of the completely skew-symmetric part, which is an axial vector defined by

$$
\chi_{\alpha}=\frac{1}{6} \varepsilon_{\alpha \beta \gamma \delta} \chi^{\beta \gamma \delta}
$$

It satisfies

$$
\chi^{\alpha}=-\frac{1}{6} \varepsilon^{\alpha \beta \gamma \delta} \chi_{\beta \gamma \delta}
$$

(the minus sign enters because of the signature of the Minkowski metric $\eta_{\alpha \beta}$ ). Note that

$$
\chi_{\alpha \beta \gamma}=\varepsilon_{\alpha \beta \gamma \delta} \chi^{\delta}, \quad \chi^{\alpha \beta \gamma}=-\varepsilon^{\alpha \beta \gamma \delta} \chi_{\delta} .
$$

Now rewrite the torsion-squared terms of the Lagrangian in terms of the irreducible parts of $\Gamma_{\alpha \beta \gamma}$. We obtain mass terms for the rotons. To first order in $\Gamma_{\alpha \beta \gamma}$,

$$
F_{\alpha \beta \gamma}=2 A_{[\alpha \beta] \gamma \gamma}, \quad F_{\alpha}=A_{\alpha},
$$


and hence

$$
\left.\begin{array}{l}
J_{1}=\psi_{\alpha \beta \gamma} \psi^{\alpha \beta \gamma}-24 \chi_{\alpha} \chi^{\alpha}+\frac{2}{3} A_{\alpha} A^{\alpha}, \\
J_{2}=\frac{1}{2} \psi_{\alpha \beta \gamma} \psi^{\alpha \beta \gamma}+24 \chi_{\alpha} \chi^{\alpha}+\frac{1}{3} A_{\alpha} A^{\alpha} \\
J_{3}=A_{\alpha} A^{\alpha} .
\end{array}\right\}
$$

The linear invariant

$$
F=2 \hat{\alpha}_{\alpha} A^{\alpha}+\frac{1}{2} \psi_{\alpha \beta \gamma} \psi^{\alpha \beta \gamma}+6 \chi_{\alpha} \chi^{\alpha}-\frac{2}{3} A_{\alpha} A^{\alpha}
$$

also provides mass terms; the first term, being a divergence, is irrelevant. Therefore, the mass term for the rotons, contained in $\mathscr{V}$, is

$$
-\frac{m^{2}}{2}\left(\psi_{\alpha \beta \gamma} \psi^{\alpha \beta \gamma}+12 \chi_{\alpha} \chi^{\alpha}--\frac{4}{3} A_{\alpha} A^{\alpha}\right)-\frac{9 \lambda}{l^{2}} \chi_{\alpha} \chi^{\alpha}
$$

where

$$
m^{2}=b-\left(1 / 2 l^{2}\right)
$$

The choice $m^{2}=0, \lambda=0$ avoids tachyons by making all the rotons massless. This particular case corresponds to the combination

$$
-\frac{e}{4 l^{2}}\left(2 F-\frac{1}{2} J_{1}-J_{2}+2 J_{3}\right)
$$

in the Lagrangian density of the full theory. The teleparallelism limit is then Einstein's theory. The spin content of the linearized version of this class of Poincare gauge theories has been analysed in detail by Kuhfuss and Nitsch (1986), who determined the conditions to be satisfied by the coefficients in the curvature-squared part of the Lagrangian, in order that linearization should lead to a viable theory of massless rotons.

Our aim is to show that non-zero values of $m^{2}$ and $\lambda$ can still give rise to a viable theory. provided that the roton modes with the wrong sign in the mass term (32) are made non-propagating by eliminating their kinetic terms. The remaining modes then describe rotons with mass.

For the convenience of the reader who wishes to compare our work with that of Kuhfuss and Nitsch, we give here the relations connecting our invariants with theirs:

$$
\begin{aligned}
& L_{1}=\frac{e}{2} J_{1}, \quad L_{2}=\frac{e}{4}\left(J_{1}-2 J_{2}\right), \quad L_{3}=\frac{e}{2}\left(J_{1}-2 J_{3}\right), \quad L_{4}=e F, \\
& L_{5}=\frac{e}{2}\left(I_{4}+I_{5}\right), \quad L_{6}=\frac{e}{2}\left(I_{5}-I_{4}\right), \quad L_{7}=\frac{e}{2}\left(I_{1}+I_{3}\right), \quad L_{8}=\frac{e}{2}\left(I_{1}-I_{3}\right) .
\end{aligned}
$$

\section{Kinetic terms for the rotons}

After some rather heavy algebra, we arrive at the following expressions for the variations of the six linearized curvature-squared invariants under variation of $\Gamma_{v \alpha \beta}$ (we 
throw out divergences):

$$
\begin{aligned}
\delta I_{1}= & \delta \psi_{v \alpha \beta}\left(-4 \square \psi^{v \alpha \beta}+8 \partial_{\mu} \partial^{\alpha}\left(\psi^{\mu \nu \beta}+\chi^{\mu \nu \beta}\right)+\frac{8}{3} \partial^{v} \partial^{\alpha} A^{\beta}\right) \\
& +\delta \chi_{v \alpha \beta}\left(-4 \square \psi^{v \alpha \beta}+4 \partial_{\mu} \partial^{\alpha}\left(\psi^{\mu \nu \beta}+\chi^{\mu \nu \beta}\right)\right) \\
& +\delta A_{\beta}\left(-\frac{16}{9} \square A^{\beta}-\frac{8}{9} \partial^{\beta} \partial_{\mu} A^{\mu}+\frac{8}{3} \partial_{\mu} \partial_{v} \psi^{\mu \nu \beta}\right), \\
\delta I_{2}= & \delta \psi_{v \alpha \beta}\left(\square \psi^{v \alpha \beta}+2 \partial_{\mu} \partial^{\alpha}\left(\psi^{\beta \mu v}-\psi^{\mu \nu \beta}\right)-\frac{2}{3} \partial^{v} \partial^{\alpha} A^{\beta}\right) \\
& +\delta \chi_{v \alpha \beta}\left(-2 \square \chi^{v \alpha \beta}+6 \partial_{\mu} \partial^{\alpha} \chi^{v \mu \beta}\right) \\
& +\delta A_{\beta}\left(\frac{4}{9} \square A^{\beta}+\frac{8}{9} \partial^{\beta} \partial_{\mu} A^{\mu}-\frac{2}{3} \partial_{\mu} \partial_{v} \psi^{\mu \nu \beta}\right), \\
\delta I_{3}= & \delta \psi_{v \alpha \beta}\left(-8 \partial_{\mu} \partial^{\alpha}\left(\psi^{\beta \mu \nu}+\chi^{\beta \mu \nu}\right)\right) \\
& +\delta \chi_{v \alpha \beta}\left(-8 \partial_{\mu} \partial^{\alpha}\left(\psi^{\beta \mu \nu}+\chi^{\beta \mu \nu}\right)\right) \\
& +\delta A_{\beta}\left(-\frac{8}{3} \partial^{\beta} \partial_{\mu} A^{\mu}\right), \\
\delta I_{4}= & \delta \psi_{v \alpha \beta}\left(-2 \partial^{\alpha} \partial_{\mu}\left(\psi^{\beta \mu \nu}+\chi^{\beta \mu \nu}\right)\right) \\
& +\delta \chi_{v \alpha \beta}\left(-2 \partial^{\alpha} \partial_{\mu}\left(\psi^{\beta \nu \mu}+\chi^{\beta \mu v}\right)\right) \\
& +\delta A_{\beta}\left(-\frac{8}{3} \partial^{\beta} \partial_{\mu} A^{\mu}\right), \\
\delta I_{5}= & \delta \psi_{v \alpha \beta}\left(-2 \partial^{\alpha} \partial_{\mu}\left(\psi^{\nu \mu \beta}+\chi^{v \mu \beta}\right)+\frac{4}{3} \partial^{v} \partial^{\alpha} A^{\beta}\right) \\
& +\delta \chi_{v \alpha \beta}\left(-2 \partial^{\alpha} \partial_{\mu}\left(\psi^{v \mu \beta}+\chi^{v \mu \beta}\right)\right) \\
& +\delta A_{\beta}\left(-\frac{8}{9} \square A^{\beta}-\frac{16}{9} \partial^{\beta} \partial_{\mu} A^{\mu}+\frac{4}{3} \partial_{\mu} \partial_{v} \psi^{\mu \nu \beta}\right), \\
\delta I_{6}= & \delta A_{\beta}\left(-8 \partial^{\beta} \partial_{\mu} A^{\mu}\right) .
\end{aligned}
$$

We now determine the conditions that have to be imposed on the constants $a_{1}, \ldots, a_{6}$ in order that the linear equations for $\psi_{v \alpha \beta}, \chi_{v \alpha \beta}$ and $A_{\beta}$ shall be decoupled. The requirements that $\chi_{v \alpha \beta}$ and $A_{\beta}$ shall not appear in the Euler-Lagrange equation for $\psi_{v \alpha \beta}$ are equivalent, respectively, to the conditions

$$
\begin{aligned}
& 4\left(a_{1}-a_{3}\right)-a_{4}+a_{5}=0, \\
& 4 a_{1}-a_{2}+2 a_{5}=0 .
\end{aligned}
$$

We find that (36) is also the condition that $\psi_{v \alpha \beta}$ shall not appear in the equation of $\chi_{v \alpha \beta}$ and (37) is the condition that $\psi$ shall not occur in the equation of $A_{\beta}$.

Now when we look for the condition that the Euler-Lagrange equation of $\psi_{v \alpha \beta}$ shall be a massive spin- 2 equation without spin 1 and spin 0 components, and the condition that the equation for $\chi_{\nu \alpha \beta}$ shall be pure spin-zero, a remarkable 'coincidence' emerges: we find just the same two conditions (36) and (37).

The $\psi$-equation is given by

where

$$
\delta \psi_{v \alpha \beta}\left(-k_{0} \square \psi^{v \alpha \beta}-\partial_{\mu} \partial^{\alpha}\left(k_{1} \psi^{\mu \nu \beta}+k_{2} \psi^{\beta \mu \nu}\right)-m^{2} \psi^{v \alpha \beta}\right)=0,
$$

$$
\left.\begin{array}{l}
k_{0}=4 a_{1}-a_{2}, \\
k_{1}=8 a_{1}+2 a_{2}+2 a_{5}, \\
k_{2}=-2 a_{2}+8 a_{3}+2 a_{4}+2 a_{5} .
\end{array}\right\}
$$


After picking out the appropriate irreducible part of the second term, we find the EulerLagrange equation

$$
\begin{aligned}
k_{0} & \square \psi^{\nu \alpha \beta}+\left(\frac{1}{6} k_{1}+\frac{1}{2} k_{2}\right) \partial_{\mu}\left(\partial^{\alpha} \psi^{\mu \nu \beta}-\partial^{\beta} \psi^{\mu \nu \alpha}\right) \\
& +\frac{1}{2} k_{2} \partial_{\mu}\left(\partial^{\alpha} \psi^{\beta \mu \nu}-\partial^{\beta} \psi^{\alpha \mu v}\right)-\frac{1}{3}\left(k_{1}-\frac{1}{2} k_{2}\right) \partial_{\mu} \partial^{v} \psi^{\mu \beta \alpha} \\
& -\frac{k_{1}}{6} \partial_{\rho \sigma}\left(\eta^{\nu \alpha} \psi^{\rho \sigma \beta}-\eta^{\nu \beta} \psi^{\rho \sigma \alpha}\right)+m^{2} \psi^{\nu \alpha \beta}=0 .
\end{aligned}
$$

Spin- 1 is eliminated if this equation implies $\partial_{v} \psi^{v \alpha \beta}=0$. Taking the divergence, we find

$$
\begin{aligned}
& \left(k_{0}+\frac{1}{3}\left(k_{1}-\frac{1}{2} k_{2}\right)\right) \square \partial_{v} \psi^{v \alpha \beta}+\frac{1}{6} k_{2} \partial_{\mu} \partial_{\nu}\left(\partial^{\alpha} \psi^{\mu \nu \beta}-\partial^{\beta} \psi^{\mu \nu \alpha}\right) \\
& \quad+m^{2} \partial_{v} \psi^{\nu \alpha \beta}=0 .
\end{aligned}
$$

The required conditions are therefore $k_{0}+\frac{1}{3}\left(k_{1}-\frac{1}{2} k_{2}\right)=0$ and $k_{2}=0$. They are just the conditions (36) and (37)! The equation of $\psi_{v \alpha \beta}$ is now

$$
\begin{aligned}
& \square \psi^{v \alpha \beta}-\frac{1}{2} \partial_{\mu}\left(\partial^{\alpha} \psi^{\mu v \beta}-\partial^{\beta} \psi^{\mu v \alpha}\right)+\partial_{\mu} \partial^{v} \psi^{\mu \beta \alpha} \\
& +\frac{1}{2} \partial_{\rho \sigma}\left(\eta^{v \alpha} \psi^{\rho \sigma \beta}-\eta^{\nu \beta} \psi^{\rho \sigma \alpha}\right)+\frac{m^{2}}{2 a_{5}} \psi^{\nu \alpha \beta}=0 .
\end{aligned}
$$

Define

$$
\psi^{v \alpha}=\partial_{\beta} \psi^{v \alpha \beta}
$$

Then, because of the symmetry properties of $\psi^{v \alpha \beta}$, and the divergence condition $\partial_{v} \psi^{v \alpha \beta}$ $=0$, we find that $\psi^{v \alpha}$ is symmetric. Because $\psi^{v \alpha \beta}$ is traceless, we find that $\psi^{v \alpha}$ is traceless and divergenceless. The Euler-Lagrange equation (42) implies a Klein-Gordon equation on $\psi^{v \alpha}$. We then get the standard massive spin-2 free field theory, in the form

$$
\left.\begin{array}{l}
\psi^{\alpha \beta}=\psi^{\beta x}, \\
\psi_{\alpha}^{\alpha}=0, \\
\square \psi^{\alpha \beta}-\frac{m^{2}}{3 a^{5}} \psi^{\alpha \beta}=0 .
\end{array}\right\}
$$

Another traceless symmetric rank two field with opposite parity is obtained from the dual of $\psi^{v \alpha \beta}$, in the same way that $\psi^{v \alpha}$ was obtained from $\psi^{v \alpha \beta}$. It satisfies the same equations. Ghosts and tachyons are avoided provided

$$
m^{2}>0, \quad a_{5}<0
$$

The Euler-Lagrange equation for $\chi_{v \alpha \beta}$ is given by

$$
\delta \chi_{\nu \alpha \beta}\left(-\beta_{0} \square \chi^{\nu \alpha \beta}-\beta_{1} \partial_{\mu} \partial^{\alpha} \chi^{\mu \nu \beta}-n^{2} \chi^{\nu \alpha \beta}\right)=0,
$$

where

$$
\begin{aligned}
& \beta_{0}=4 a_{1}+2 a_{2} \\
& \beta_{1}=4 a_{1}+6 a_{2}+8 a_{3}+2 a_{4}-2 a_{5}
\end{aligned}
$$

and

$$
n^{2}=12 m^{2}+\left(18 \lambda / l^{2}\right)
$$


More explicitly, the Euler-Lagrange equation is

$$
\beta_{0} \square \chi^{v \alpha \beta}+\frac{1}{3} \beta_{1} \partial_{\mu}\left(\partial^{\alpha} \chi^{\mu v \beta}+\partial^{\beta} \chi^{\mu \alpha \nu}+\partial^{v} \chi^{\mu \beta \alpha}\right)+n^{2} \chi^{v \alpha \beta}=0 .
$$

The spin- 1 component will be absent provided that this equation implies $\partial_{v} \chi^{v a \beta}=0$. The required condition is $\beta_{1}=3 \beta_{0}$. But this is just (36)! The equation satisfied by the axial vector $\chi_{\alpha}$ turns out to be simply

$$
\beta_{0} \partial_{\alpha} \partial_{\mu} \chi^{\mu}+n^{2} \chi_{\alpha}=0
$$

The divergence condition on $\chi_{v \alpha \beta}$ is equivalent to

$$
\partial_{\mu} \chi_{\nu}-\partial_{\nu} \chi_{\mu}=0
$$

so that $\chi_{\mu}=\partial_{\mu} \chi$ for some pseudo-scalar $\chi$, satisfying the Klein-Gordon equation

$$
\square \chi+\left(n^{2} / \beta_{0}\right) \chi=0
$$

Ghosts and tachyons are avoided provided

$$
n^{2}>0, \quad \beta_{0}>0
$$

The Euler-Lagrange equation for $A_{\mu}$ is

$$
\frac{8}{9}\left(a_{1}-a_{2}+3 a_{3}+3 a_{4}+2 a_{5}+9 a_{6}\right) \partial^{\beta} \partial_{\mu} A^{\mu}-\frac{4}{3} m^{2} A^{\mu}=0 .
$$

The mass term has the wrong sign. A tachyon is avoided if we eliminate the kinetic term by imposing the condition

That is,

$$
a_{1}-a_{2}+3\left(a_{3}+a_{4}\right)+2 a_{5}+9 a_{6}=0 .
$$

$$
3 a_{6}=a_{1}-a_{3}-a_{4}
$$

the Euler-Lagrange equation of $A_{\mu}$ in this linearized free-field limit is then simply $A_{\mu}=0$. In the full theory, $A_{\mu}$ is proportional to the trace of the spin-tensor of matter (compare the way that torsion in Einstein-Cartan-Kibble-Sciama theory does not propagate but is determined algebraically by the spin-tensor of matter. The ECKS theory is the very particular case in which the only non-zero coefficient in (6) is $b$ ).

The physical requirements that we have imposed are by no means sufficient to determine a unique theory. Apart from the inequalities, we have found just three conditions (36), (37) and (55) on the eight parameters $a_{1} \cdots a_{6}, b, \lambda$ (the parameter $l$ is known: it is the Planck length). Theories connected by the parameter transformation (8) are equivalent. So we have obtained a four-fold infinity of theories. Even fixing $\lambda$ and the masses of the spin 2 and spin zero rotons would still leave one free parameter. The theories belonging to such a one-parameter family are not equivalent beyond the linearized approximation; they are distinguishable in their predicted interactions among rotons.

\section{Conclusions}

We have found a set of conditions that must be satisfied by the coefficients in the Lagrangian density of a Poincaré gauge theory, in order that the linearized theory, 
interpreted as a theory of fields in a flat background space, shall describe massive 'rotons' with unique spins without ghosts or tachyon modes. We have arrived at a class of theories which all have the following features: as well as the classical graviton which is the massless $2^{+}$described by the translational gauge potential, there are also massive $2^{+}$and $2^{-}$rotons (of equal mass), as well as a massive pseudoscalar $\left(0^{-}\right)$roton.

The existence of massive spin-2 fields in Poincare gauge theories is particularly interesting in view of the successes of strong gravity theories (Sivaram and Sinha 1979) in which the consequences of the hypothesis of a short range strong component of gravity are explored.

\section{Acknowledgements}

We wish to thank P Goswami for his interest in the work and for his helpful suggestions. We also thank the University Grants Commission of India for financial support of this work.

\section{References}

Cho Y M 1976 Phys. Rev. D14 2551

Hehl F W 1980 in Proceedings of the sixth international school of cosmology and gravitation (eds) P G Bergmann and V Sabbata (New York: Plenum)

Kuhfuss R and Nitsch J 1986 Propagating modes in gauge field theories of gravity, Report no. MPA 223 Max Planck Institut fur Astrophysik, Garching

Lord E A, Sinha K P and Sivaram C 1974 Prog. Theor. Phys. 52161

Nieh H T 1980 J. Math. Phys. 211439

Nitsch J 1980 Proc. Sixth Int. School of cosmology and gratitation (eds) P G Bergmann and V Sabbata (New York: Plenum)

Sinha K P 1984 Pramana-J. Phys. 23205

Sivaram C and Sinha K P 1979 Phys. Rept. 51111 